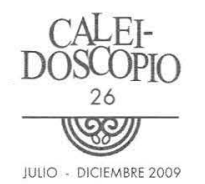

\title{
Acercamiento al indeterminismo científico
}

Fl determinismo es la postura epistémica que sostiene que todo Esto está intrínsecamente relacionado no sólo con las ideas religiosas y metafísicas, sino también con ideas científicas - principalmente en física, sobre todo aquellas que describen los fenómenos desde un punto de vista macroscópico y a bajas velocidades-. Tal punto de vista ha sido sumamente criticado, sobre todo por la postura denominada indeterminismo.

En el presente trabajo se examinará, brevemente, en qué consisten el determinismo y el indeterminismo, haciendo énfasis en el indeterminismo científico y buscando su relación con el Principio de Incertidumbre de Heisenberg, que aparece en la mecánica cuántica.

\section{INTRODUCCIÓN}

Es admisible pensar que en los comienzos de la historia humana, el hombre, para intentar explicar el acontecer natural mediante métodos observacionales aún deficientes, no tuviera más opción que acudir a la magia, los ritos y la religión, por ser lo más inmediato y accesible para abordar la ocurrencia de las cosas, aun cuando con ello 
se creyera que el mundo es gobernado por entidades poderosas que deciden qué, cuándo, cómo y para qué suceden las cosas en el mundo. Esta manera de proceder generó explicaciones insatisfactorias donde los resultados de los eventos no siempre concordaban con lo predicho. Fue precisamente esta necesidad de prever con mayor precisión los hechos venideros lo que sembró el interés por buscar una explicación del comportamiento natural. Este deseo por conocer los hechos futuros germinó de manera continua en todas las sociedades, provocando con ello una mejora sensible en las técnicas de observación, recolección, ordenamiento y análisis de los datos, condujo a que algunas civilizaciones alcanzaran grandes logros explicativos que evidenciaron la regularidad de varios fenómenos naturales, destacándose por su trascendencia, los movimientos periódicos. En este rubro destacan los babilonios y los egipcios, aunque fueron los griegos quienes hicieron de la observación un arte y, aun cuando mantenían vigente una cosmovisión mítica y teológica, fueron la reflexión y la abstracción racional los verdaderos motores de conocimiento que los llevaron a la búsqueda de una explicación de cómo son las cosas.

Con el estudio de las regularidades naturales, la idea de que el mundo está obligado a seguir determinados procesos va ganando terreno dentro de la visión cosmogónica de los distintos pueblos, y con ello, se impone lenta, pero inexorablemente, una concepción determinista de la naturaleza. Esta visión permite predecir, basándose en las condiciones actuales, lo que ocurrirá con el mundo natural en un tiempo futuro, así sea aproximadamente. Estos éxitos predictivos son los que permiten afincar el determinismo en un pensamiento científico incipiente, que emplea hipótesis experimentales como argumentos explicativos.

Ahora bien, la noción de determinismo queda plasmada en la literatura griega, en donde la vida y evolución de los personajes están perfectamente "determinadas" por la trama y las características físicas y psicológicas asignadas por el autor. Así, un lector de la obra puede comprender cómo las vidas de los personajes siguen una senda 
trazada de antemano, es decir, su vida está "determinada". Quizá el caso más conocido sea la tragedia de Edipo, quien después de conocer por el Oráculo de Delfos lo que le deparaba el destino, busca infructuosamente evadir su sino para caer, irremisiblemente, en él. Posteriormente, el vocablo se trasladó a la filosofía, la ciencia y a la esfera humana en general, donde se le vinculó con la libertad. ${ }^{1}$ Aun así, y sin tener una definición única, precisa y clara, convencionalmente admitimos "el determinismo como la doctrina según la cual todos y cada uno de los acontecimientos del universo están sometidos a las leyes naturales. Estas leyes son de carácter causal". ${ }^{2}$ Entendido el determinismo en términos de leyes naturales ha sido demasiado cuestionado, debido a la objeción que existe respecto a la validez del causalismo o a las dudas de si las leyes naturales existen realmente o no son más que una muleta que apoya al método científico, por lo que el determinismo no es más que un concepto ambiguo. Earman señala, en este sentido, que

Perhaps the most venerable of all the philosophical definitions holds that the world is deterministic just in case every event has a cause. The most immediate objection to this approach is that it seeks to explain a vague concept-determinism-in terms of a truly obscure one -causation- ${ }^{3}$

Hay que considerar, sin embargo, si efectivamente la tesis de que la naturaleza se halla sometida a ciertos patrones de comportamiento

1 Por ejemplo, Popper hace el señalamiento de que el determinismo coarta la libertad, al proferir: "Hasta casi 1927 los físicos, salvo contadas excepciones, creían que el mundo era un inmenso reloj sumamente preciso. Descartes [...] describió el reloj como algo mecánico: toda causalidad era empuje. Tal fue la primera y más clara teoría de la causalidad. Tiempo después, aproximadamente a partir de 1900, el mundo pasó a ser considerado como un reloj eléctríco. Mas en ambos casos se consideraba que el mundo era un reloj sumamente preciso. [...] En tal mundo no quedaba lugar para las decisiones humanas. Nuestras sensaciones de estar actuando, planeando, comprendiéndonos mutuamente, eran ilusorias. Pocos filósofos, con la gran excepción de Peirce, osaron disputar esta concepción determinista. En 1927, con Werner Heisenberg, la física cuántica experimentó un gran cambio. Quedó claro que procesos ínfimos convertían al reloj en algo impreciso: existian indeterminaciones objetivas." (Popper, K. (1990): Un mundo de propensiones; Ed. Tecnos. Madrid. 1992. pp. 22-23).

2 Ferrater Mora, J. (1964).pdf:Diccionario de filosofía. Tomo I: A-K; Ed. Sudamericana. Buenos Aires. p. 418 .

3 Earman, J. (1986).pdf: A primer on determinism; D. Reidel Publishing Company; Netherlands. p. 5 
que dan pie a las regularidades observadas en la naturaleza (las regularidades estadísticas) admite una justificación plausible, bien sea desde el punto de vista de la física o no, pues es difícil argumentar tanto a favor del determinismo como en contra de él. En todo caso, Psillos aduce:

\footnotetext{
Determinism: Intuitively, the view that the past uniquely determines the future. Laplace characterized determinism as lawful predictability. [...] Freed from the epistemic notion of predictability, determinism is taken to be a claim about universal causation: each and every event has a fully sufficient nomological condition (i.e., a sufficient cause in accordance with universal laws). Determinism, then, denies the existence of objective chance in the world: all events are determined to happen with either probability one or zero. ${ }^{+}$
}

No hay, por tanto, una definición única para el determinismo, aunque este autor también resalta que dicho término descansa en la relación causal y converge hacia las leyes naturales, las cuales se introducen mediante un proceso inductivo derivado de la observación de cómo un conjunto de eventos naturales se comporta, aproximadamente, de manera similar bajo las mismas circunstancias. Esta regularidad surgida de la observación se eleva a rango de ley y se asume, en principio, como evidente para todos los observadores. Dicha ley, en términos concisos y puntuales, no es más que la expresión verbal que enuncia la regularidad estadística llevada al límite, es decir,

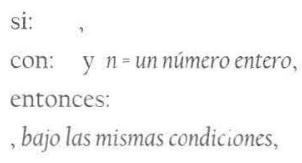

con lo que se enuncia la constancia y repetitividad de un suceso de manera (aproximadamente) idéntica a la ocurrencia de los sucesos previos, modificando su estado de ocurrencia sólo bajo la acción, deliberada o accidental, de un agente-sea externo o no- que no se ciña a las condiciones estipuladas.

4 Psillos, S. (2007).pdf: Philosophy of science A-Z; Edinburgh University Press; G. B. p. 67. 
Ahora bien, la regularidad estadística tiene dos vertientes: por un lado, nos lleva a un determinismo causalista, que es aquél en que si después de considerar un número muy grande de observaciones bajo condiciones constantes e iguales, notamos que éstas son estables o se mantienen sin variabilidad estadística significativa, decimos que tenemos una probabilidad de ocurrencia unitaria o un evento certero. ${ }^{5}$ En caso de que esto no se satisfaga, es decir, si se presenta variabilidad estadística significativa, podremos hablar de un determinismo probabilista.

Así, parece claro que las leyes naturales son extrapolaciones justificadaslógicamentemedianteunprocesodeinducciónqueposeen un contenido de verdad no nulo sustentado en evidencias empíricas y que permiten establecer principios generales y fundamentales que vinculan lo observado individualmente con el conjunto de eventos similares. Es decir, asumimos que al observar un evento que se ha comportado de una manera particular bajo determinadas condiciones, entonces las observaciones de la misma clase de eventos, bajo las mismas condiciones, se comportarán de la misma manera. Esta generalización, y simplificación, del comportamiento de los sucesos nos permite representar simbólicamente el cúmulo

5 Un fenómeno aleatorio, fortuito o al azar, es un fenómeno empírico que se caracteriza por la propiedad de que, al observarlo bajo determinado conjunto de condiciones, no siempre se obtiene el mismo resultado, es decir, no existe regularidad determinista, sino que los diferentes resultados ocurren con regularidad estadística; esto lleva a decir que existen números entre los valores 0 y 1 que representan la frecuencia relativa con la que se observan los diferentes resultados en una serie de repeticiones independientes del fenómeno. Decimos entonces que la probabilidad de que ocurra el evento $A$, denotada por $\mathrm{P}(\mathrm{A})$, con $\mathrm{A}$ elemento de un espacio muestral $\Omega$, es un valor que se encuentra entre los valores 0 y 1 . En términos probabílisticos, la certeza -o evento seguro- está dado por $\mathrm{P}(\Omega)=1$, mientras que el evento imposible está dado por $P(A)=0$. Más aún, si se tiene una serie muy grande de pruebas, se observa que el evento favorable puede producirse con una frecuencia próxima a su probabilidad (ley empírica del azar); así que, un suceso cuya probabilidad no difiere de l sino en una cantidad extremadamente pequeña $\left(=10^{-100}\right)$, puede considerarse, efectivamente, como humanamente cierto. Sin embargo, aquí no hay que perder de vista que las cuestiones estadísticas siempre son problemas de las probabilidades de las causas. En forma más clara, Borel arguye "... Es necesario decir alounas palabras acerca de la forma en que se plantean, con frecuencia, los problemas prácticos, siendo de la forma: "tal resultado, ¿se debió al azar o tuvo alguna causa? A esta pregunta, la teoría de probabilidades proporciona a menudo una respuesta que no habría que considerar como decisiva, pero que sería excesivo negarle todo valor". Borel, E. (1996); Probabilidad y Errores; Colección Metronómica, Serie Metrología Técnica, Limusa Noriega Ed.; México. p 98.

C A L E


de experiencias similares mediante expresiones relacionales que se orientan a dar una 'explicación' basada en la lógica, lo cual nos conduce a interpretar los resultados introduciendo la idea de que tal comportamiento se dio así porque había algo que lo orilló a comportarse de esa forma y no de otra, y que lo mismo ocurrirá con todos y cada uno de los eventos que se sometan a observación. En esto consisten las leyes naturales, es decir, son simplificadoras metodológicas, y al ser susceptibles de verificación experimental, se emplean como justificantes de la relación causal, brindándonos la posibilidad de seguir, en términos funcionales, el comportamiento del sistema en todo tiempo, y proporcionándonos la enorme ventaja de pronosticar y comprender la evolución del sistema al volverlo visualizable e, incluso, lógicamente justificable. Claramente, en el caso presente estamos refiriéndonos a las leyes naturales como enunciados observacionales cimentados en la relación causal como motor de ocurrencia y como regla universal. Bird clarifica esto cuando dice que

\footnotetext{
Laws need to be distinguished from statements of laws and from our theories about what laws there are. Laws are things in the world which we try to discover. In this sense they are facts or are like them. A statement of a law is a linguistic item and so need not exist, even though the corresponding law exists (for instance if there were no people to utter or write statements). Similarly, theories are human creations but laws of nature are not. The laws are there whatever we do-one of the tasks of the scientist is to speculate about them and investigate them. A scientist may come up with a theory that will be false or true depending on what the laws actually are. And, of course, there may be laws about which we know nothing. ${ }^{6}$
}

Y es que legislar el comportamiento de los sistemas naturales es el primer paso que se debe dar para comprender, y posteriormente explicar, la ocurrencia de los sucesos, pues sólo nos abocamos a analizar las regularidades por ser ellas las que se pueden modelar. Los eventos azarosos, en tanto que únicos e irrepetibles no pueden modelarse, quedando sólo como un caso anecdótico o curioso al ser un evento aislado. Max Planck, por su parte, señala:

6 Bird, A. (1998): Philosophy of Science; Fundamentals of Philosophy. Series Editor: John Shand. University of Edinburgh. Routledge. Taylor \& Francis Group. pdf. p. 17. 
¿Cómo descubrimos las leyes de la Física y cuál es su naturaleza? Debe ser remarcado, para empezar, que no tenemos ningún derecho de asumir que alguna ley física existe, o que si ha existido hasta ahora, existirá para siempre en el futuro. Es perfectamente concebible que un buen día la Naturaleza producirá un evento que nos sorprenderá a todos; y si esto llegara a pasar estaríamos a merced de tal evento, aun si el resultado fuera tal que, a pesar de nuestros empeños, falláramos en introducir orden en la confusión restante. En tal evento, la única ruta abierta a la ciencia sería declararse en bancarrota. Por esta razón, la ciencia es impelida a empezar por la suposición muy general de que una regla general domina a través de toda la naturaleza. ${ }^{7}$

Es fácilmente entendible entonces por qué el determinismo, y quizá aún más la relación causal, germinó en el pensamiento científico como el mecanismo explicativo por excelencia, a tal grado que ha sido, en términos generales, parte fundamental de la física clásica. De hecho, fue la física quien impulsó el uso generalizado de la relación causal como un elemento necesario para explicar los sucesos del mundo natural. La visión del mundo como un dispositivo mecánico se impuso gracias a las simplificaciones conceptuales y matemáticas que Newton introdujo en la física para explicar el comportamiento de los objetos; estas suposiciones simplificadoras que aparecieron en la axiomatización de las leyes físicas le confirieron a los fenómenos una simplicidad asombrosa que sólo requería de un conector entre la causa y el efecto; dicho conector fue la fuerza. Otra ventaja que se encontró al introducir la fuerza como elemento explicativo fue que el uso de este concepto era el mismo para todos los observadores, pues debían llegar a resultados equivalentes al estar sometidos al requerimiento de un tiempo y un espacio considerados absolutos. Esto posibilitó que los eventos se matematizaran, y con esta representación simbólica, se pudo predecir y retrodecir el estado del sistema en cualquier instante, necesitando conocerse únicamente las condiciones iniciales, pudiendo ser éstas fijas o aleatorias. Esta visión de la ciencia satisfacía el ideal platónico de representar la realidad matemáticamente, y concordaba con las aspiraciones de Galileo, de Descartes y de Kepler. Así, el determinismo parecía imponerse

7 Félix Valdez, J. (2001): La mecánica cuântica. 1 $1^{a}$ Ed.; Colección Nuevo Siglo. Serie Ciencia y Tecnología. Universidad de Guanajuato. SEP. 2001. p. 99. 
sobre una naturaleza caótica, imponiendo un orden en todos y cada uno de los elementos del mundo.

\section{EL DETERMINISMO LAPLACIANO}

El personaje más connotado del determinismo científico es Laplace, que empleóla mecánica newtoniana para explicar el comportamiento de los cuerpos celestes. Laplace fincó la relación causal en la idea de fuerza, considerando que tal concepto puede interpretarse como el equivalente al eslabón de una sucesión de eventos que aparecen en orden secuencial, donde la causa y el efecto asumirían el papel de piñones en contacto y donde (aparentemente) se cuenta con una dirección preferencial de avance. El concepto fuerza, propuesto por Newton en el siglo XVII, fue el principal baluarte de la física hasta los albores del siglo XX, que fue cuando apareció la mecánica cuántica. Al apoyarse en este concepto físico-matemático, Laplace apologiza tenazmente la relación causal dentro de la naturaleza, pues para él

Todos los sucesos, hasta aquellos que a causa de su insignificancia no parecen seguir las grandes leyes de la naturaleza, son el resultado de ellas tan exacta y necesariamente como las revoluciones del sol [... Supongamos por un instante el caso de una inteligencia que pudiera abarcar todas las fuerzas en virtud de las cuales la naturaleza está animada y la situación respectiva de los seres que la componen -una inteligencia suficientemente vasta para someter a análisis estos datos-, ella debería comprender en la misma fórmula los movimientos de los mayores cuerpos del universo y los movimientos del más ligero átomo; para ella, nada sería incierto y el futuro y el pasado serían el presente a sus ojos. ${ }^{8}$

Laplace expuso que si se conoce el estado actual de un sistema, es dable predecir con una precisión elevada cualesquier otro estado futuro de ese sistema. Esto lo llevó a decir que a todo efecto le antecede una causa, relación conocida como relación de causa-efecto. Es altamente significativa la formulación que aparece en su Essai Philosophique sur les Probabilités, de 1819:

8 Hacking, I. (1995); La domesticación del azar (La erosión del determinismo y el nacimiento de las ciencias del caos); Ed. Gedisa. España. p. 32. 
Debiéramos...considerar el presente estado del universo como el efecto de su estado anterior y la causa del que seguirá. Supongamos...una inteligencia que pudiera conocer todas las fuerzas que animan a la naturaleza, y los estados, en un instante, de todos los objetos que la componen... para [esta inteligencia] nada podría ser incierto; y el futuro, como el pasado, sería presente a sus ojos. ${ }^{9}$

En este parágrafo hasta cierto punto eufórico, Laplace ${ }^{10}$ extrapola el determinismo y lo hace yacer en las entrañas de la naturaleza. Al igual que Newton, Laplace exalta una deidad, pues bajo su concepción determinista, parece ser evidente que tal "inteligencia" o potestad superior es quien rige la naturaleza y es capaz de fusionar el pasado y el futuro en un instante pudiendo, por tanto, predecir el ulterior comportamiento del sistema, es decir, implícitamente enuncia que en todo fenómeno natural la causa debe ser origen y/o motivo del consecuente efecto. Esto será viable siempre y cuando los sistemas físicos sean inmutables en el tiempo, haciendo con ello posible conocer con absoluta certeza el comportamiento futuro de un evento a partir del conocimiento de cualquier estado anterior y del conocimiento de las leyes naturales.

No es raro entonces que al observar fenómenos repetitivos, se buscase una explicación científica de lo ocurrido con silogismos convincentes y efectivos que tornaran la explicación en algo sencillo y claro. Las leyes naturales que la mecánica newtoniana exalta terminan por posicionar en un sitio de honor el determinismo a lo largo de doscientos años y simultáneamente sirven para desproveer del animismo el acaecer de los fenómenos en la naturaleza. Esta basificación de la relación causal en la teoría se asume como el principio más elemental del mundo a la vez que se erige también como el elemento que articula y estructura bien sea los conceptos teóricos o los procesos empíricos, además de introducir en la ciencia una metodología que es, hasta cierto punto, rígida pero efectiva. Con esto se alcanza a cumplir una

9 Tomado de: Popper, K. R. (1996): El universo abierto. Un argumento a favor del indeterminismo. Post Scriptum a la Lógica de la Investigación Cientifica. Vol. II.; Tecnos. España. p. 22.

10 No se debe perder de vista que Laplace es uno de los pioneros de la Probabilidad y la Estadística. 
de las aspiraciones de los filósofos griegos pues, como Martínez expone,

Otros filósofos importantes en el desarrollo de esta tradición teórica son Pitágoras, Anaxágoras y Demócrito. De una manera diferente, cada uno de ellos elaboró la idea central de las tradiciones teóricas: el rechazo a factores explicativos sobrenaturales y la búsqueda de principios explicativos naturales de carácter general que permitieran dar cuenta de la experiencia de una manera inteligible. Estos filósofos no estaban interesados en la sistematización de datos de la experiencia como un fin importante de por sí, sino más bien en la función que esta sistematización ejercía en discusiones abstractas en las que contaban la economía y la consistencia de los argumentos, así como los principios en los que se basaban las teorías. ${ }^{.1}$

Así, en el determinismo laplaciano se destacan como atributos esenciales no sólo la capacidad de predecirunívocamenteel acontecer de los eventos en el universo, sino también la manera en que la predicción descansa totalmente en lo necesario de la ocurrencia. Esto debido a que el hombre, al desconocer cuál es la estructura real de la naturaleza se ve impelido, de acuerdo con su vocación inquisitiva y racional, a imponer una condición lógica (necesaria y suficiente) a la ocurrencia de los eventos, obligando a que la causa anteceda, a fortiori, a la aparición del efecto y a que éste acontezca sólo si se dio antes aquélla; esto, como se ha mencionado líneas arriba, sólo fue posible intuirlo gracias a la sencillez y fortalezas intuitiva y conceptual del concepto fuerza.

Para Laplace, el mundo esta constituido por corpúsculos interactuando entre sí de acuerdo a las leyes de la mecánica newtoniana. Así, si se quisiera conocer el estado ${ }^{12}$ del sistema en cualquier instante $t$, tendrían que conocerse completamente sus condiciones iniciales; esto permitiría predecir el comportamiento del sistema en cualesquier tiempo posterior $t_{p}$ puesto que se satisface que: $t<t_{1}<\ldots<t_{n}$. Evidentemente, el número de variables involucradas puede ser incuantificable (por ser un sistema de

11 Martínez, Sergio F. (2001): De los efectos a las causas. Sobre la historia de los patrones de explicación científica; UNAM-Paidós. p. 30.

12 Por estado del sistema significamos el conocimiento de todas las variables necesarias para la descripción del sistema, por ejemplo: posición, masa, velocidad, dirección del movimiento, fuerza, presión, temperatura, etc. 
partículas) y la labor descriptiva de la evolución del sistema sería un trabajo sobrehumano. Laplace imaginó entonces a un demonio -como una inteligencia superior al hombre-capaz de averiguar el conjunto completo de las condiciones del sistema en cualquier instante. Así, con las condiciones iniciales y las leyes naturales, y apoyándose en las ecuaciones de la mecánica, el demonio podría deducir todos los estados futuros del sistema. Este experimento mental de Laplace exhibe que, de conocerse cabalmente las leyes de la naturaleza, entonces el futuro del sistema estaría implícito en cualquier instante de su pasado; esto es precisamente una manera de concebir al determinismo. Ahora bien, el demonio laplaciano, metafóricamente hablando, sería un científico que trabaja con un sistema, conoce sus condiciones iniciales y las teorías que gobiernan su comportamiento, más que una deidad. Popper concibe al demonio en los mismos términos:

El punto crucial del argumento de Laplace es éste: Hace de la doctrina del determinismo una verdad de la ciencia y no de la religión. El demonio de Laplace no es un Dios omnisciente, sino simplemente un supercientífico. No se le supone capaz de nada que no puedan hacer los cientificos humanos, o al menos que no puedan hacer aproximadamente: simplemente se le supone capaz de realizar sus tareas con perfección sobrehumana. ${ }^{13}$

El mismo Popper señala que las teorías que "respondan plenamente al propósito de Laplace pueden ser denominadas «deterministas prima facie»", ${ }^{14}$ donde

una teoría es determinista prima facie si, y sólo si, nos permite deducir -a partir de una descripción matemáticamente exacta del estado inicial de un sistema físico cerrado que se describe en términos de la teoría, la descripción, con cualquiera que sea el grado finito de precisión estipulado, del estado del sistema en cualquier instante dado del futuro..$^{15}$

Asimismo, la idea del determinismo puede vislumbrarse más

13 Popper, K. R. (1996): El universo abierto. Un argumento a favor del indeterminismo. Post Scriptum a la Lógica de la Investigación Cientifica. Vol. II.; Tecnos. España. p. 53.

14 Ibid. p. 54.

15 Íbid. p. 54. 
fácilmente, de acuerdo a la sugerencia de Popper, con la ayuda de la película que muestra los estados sucesivos del mundo. Tomando esto en cuenta,

Podríamos decir que el determinismo "científico" es consecuencia del intento de sustituir la vaga idea de conocimiento anticipado del futuro por la idea más precisa de predictibilidad de acuerdo con los procedimientos científicos racionales de predicción. Es decir, el determinismo afirma que el futuro puede deducirse racionalmente a partir de las condiciones iniciales pasadas o presentes en unión de teorías universales verdaderas. ${ }^{16}$

Así, este filósofo propone que el determinismo cientifico se puede definir como

la doctrina que dice que el estado de cualquier sistema físico cerrado en cualquier instante futuro dado puede ser predicho, incluso desde dentro del sistema, con cualquiera que sea el grado estipulado de precisión, mediante la deducción de la predicción a partir de teorías, en conjunción con condiciones iniciales cuyo grado de precisión requerido puede calcularse siempre (de acuerdo con el principio de poder dar razón) si la tarea de predicción es dada. ${ }^{17}$

\section{EL INDETERMINISMO}

El determinismo se relaciona estrechamente con una concepción mecanicista del universo, al considerar que la naturaleza posee un comportamiento similar al de una máquina. Históricamente, se puede considerar que el determinismo científico surge como una respuesta de los físicos para eliminar la idea de las leyes divinas a la hora de explicar cómo ocurren los eventos naturales, lo que les condujo a proponer las leyes naturales. Pero en el caso de un suceso natural, pareciera que todo sigue un guión definido de antemano, por lo que es fácil pensar que la naturaleza está sujeta a determinadas leyes naturales que gobiernan el devenir de los acontecimientos de la naturaleza, y que pueden ser descubiertas por medio de la experiencia y del razonamiento humano. Para el determinismo, si conocemos las leyes de la naturaleza, podemos predecir el futuro

16 Íbid. p. 55.

17 Ibid. p. 59. 
a partir de los datos presentes por métodos puramente racionales, dado que todo suceso en el universo está predeterminado.

¿Pero qué ocurre si hubiese un suceso que no esté predeterminado? ¿Habría que rechazar el determinismo por eso?, es decir, ¿el determinismo deja de ser válido? Es de pensar que si hubiese un sólo suceso futuro en el universo que no pudiera predecirse, o si se encontrara un sitio donde las leyes naturales dejaran de valer, habría que rechazarlo en principio, y buscar por qué no se cumplen las leyes naturales.

Hume, al indagar sobre la Teoría de las ideas, cae en este tipo de problemas, llegando a cuestionar la relación causa-efecto. Al reflexionar sobre las creencias que la gente tiene acerca de los hechos y de su percepción temporal, centra su atención en la actuación y la deliberación, y en los probables cursos de acción posibles: puesto que las acciones aún no han ocurrido, sus consecuencias tampoco se han presentado, por lo que cualquier creencia que sobre ellas se tenga será incierta y deberá referirse, necesariamente, a algo "ausente". Propone entonces:

Si se preguntara a un hombre por qué cree en un algún hecho que no presencia-por ejemplo, que su amigo está en el campo o en Francia-, daría una razón; y esta razón sería algún otro hecho, tal como una carta que recibiera de él, o el conocimiento de sus propósitos y promesas anteriores [...] Todos nuestros razonamientos relativos a hechos son de la misma naturaleza. Y en ellos se supone constantemente que hay una conexión entre el hecho presente y aquel que se infiere de él. Si no hubiera nada que los uniera, la inferencia sería totalmente incier ta. ${ }^{18}$

Debido a esto, creemos que hay un vínculo entre el hecho que observamos y la situación de lo no observado al presente, infiriendo así una cosa de la otra. Inicialmente, Hume cree que esto se debe a "inferencias causales" o razonamientos fundados en una relación causal. Para comprender mejor esa relación, propone:

Es imposible razonar de un modo correcto, sin comprender perfectamente la idea sobre la cual

18 Stroud, B. (1995); Hume; Colección Filosofía Contemporánea; Instituto de Investigaciones Filosóficas, UNAM; México. p. 66. 
razonamos; y es imposible comprender perfectamente alguna idea, sin seguirla hasta su origen y examinar la impresión primaria de la cual surge. ${ }^{19}$

Al buscar más detenidamente el origen de la idea de causalidad, se da a la tarea de indagar qué son la causa y el efecto:

En primer lugar, encuentro que cualesquiera objetos considerados como causa o efectos son contiguos, y que nada puede actuar en un tiempo o en un espacio separado, por poco que sea, de los que corresponden a su existencia. Aunque a veces parezca que objetos distantes pueden producirse unos a otros, al examinarlos se halla, por lo común, que están conectados por una cadena de causas, las cuales son contiguas entre sí y respecto de los objetos distantes; y si en algún caso particular no podemos descubrir esta conexión, suponemos empero que existe. ${ }^{20}$

Es claro que Hume supone, en primera instancia, que hay una causalidad presente en el acontecer natural, y que todas las inferencias que nos llevan de lo observado a lo no observado tienen como sustento una relación causal, es decir, que si ocurre un evento C (de causa), entonces ocurrirá un evento $\mathrm{E}$ (de efecto); o dicho en la manera habitual: todo evento debe tener una causa. Sin embargo, al profundizar en sus reflexiones, se percata de que no necesariamente se cumple que si $E$ ha acontecido es porque $C$ ha ocurrido. Es este razonamiento el que pone en tela de juicio la validez del principio de causalidad, al no encontrar alguna justificación para su generalización, asegurando

de que todos nuestros razonamientos relativos a causas y efectos no se derivan más que de la costumbre; y de que la creencia es más propiamente un acto de la parte sensitiva de nuestra naturaleza, que un acto de la parte cogitativa. Acaso al final saldrá a la luz que la inferencia no depende de la conexión, sino que la conexión necesaria depende de la inferencia. ${ }^{21}$

Esta conclusión lógica es tajante y demoledora, siendo -para Humeúnicamente la observación reiterada de los fenómenos naturales, y su desenvolvimiento por etapas (o conjunción, como la denomina) lo que nos lleva a interir la idea de la causalidad o de conexión necesaria.

\footnotetext{
19 Íbid. p. 66.

20 Íbid. p. 67.

21 Hume, cit. en Stroud, B., pp. 114 - 115.
} 


\title{
EL INDETERMINISMO CIENTÍFICO
}

La reacción a las posturas deterministas es lo que suele conocerse con el nombre de indeterminismo. Esta doctrina niega que todo lo que sucede en la naturaleza tenga una causa, además de que pueden tenerse acontecimientos sin que esté involucrada la necesariedad de la ocurrencia.

Ya se tenía como antecedente del indeterminismo la conclusión de Hume y su impecable desarrollo lógico, que resultó ser una losa muy pesada y difícil de mover para los defensores del determinismo. De hecho, tal conclusión es una de las razones que Popper esgrime siempre para atacar al determinismo científico:

\begin{abstract}
Hume estaba interesado por la condición del conocimiento humano o, como él diría, por el problema de si nuestras creencias (o, al menos, algunas de ellas, se pueden justificar con razones suficientes. Planteó dos preguntas, una lógica $\left(\mathrm{H}_{\mathrm{L}}\right)$ y otra psicológica $\left(\mathrm{H}_{\mathrm{Ps}}\right)$, con la característica importante de que sus respuestas chocan entre sí de algún modo. La pregunta lógica es la siguiente: $H_{L}$ ¿Cómo se justifica que, partiendo de casos (reiterados) de los que tenemos experiencia, lleguemos mediante el razonamiento a otros casos (conclusiones) de los que no tenemos experiencia? [...] La pregunta psicológica es la siguiente: $\mathrm{H}_{\mathrm{ps}}$ : :Por qué, a pesar de todo, las personas razonables esperan y creen que los casos de los que no tienen experiencia van a ser semejantes a aquellos de los que tienen experiencia? Es decir, ¿Por qué confiamos tanto en las experiencias que tenemos? ${ }^{22}$
\end{abstract}

Las respuestas que Hume da a las preguntas anteriores ya las conocemos: no existe ninguna justificación, salvo la costumbre. Pero Popper continúa exponiendo argumentos que apuntan directamente al centro de flotación del determinismo científico, al esgrimir que nuestras teorías no son más que "redes creadas por nosotros para atrapar el mundo... Las teorías no son sólo instrumentos. A lo que aspiramos es a la verdad". ${ }^{23}$ Por ello, Popper propone un argumento lógico a favor del indeterminismo: la imposibilidad de la autopredicción. En él, arguye el filósofo, si poseyéramos un

22 Popper, K. R. (1982): Conocimiento Objetivo. Un enfoque evolucionista; Ed. Tecnos. Madrid. pp. 1718.

23 Ibíd., Poper. El Universo abierto. p. 65.

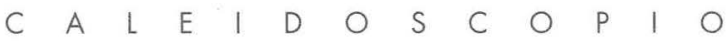


conocimiento teórico perfecto, y bien definidas las condiciones iniciales, se podría predecir, deductivamente, los estados futuros de cada persona. Pero esto es absurdo, puesto que nos llevaría a una teoría con contradicciones internas. ${ }^{24}$

Pero igualmente, cuando se estudia el determinismo laplaciano y se intenta justificar, se encuentran algunas dificultades, pues la naturaleza no exhibe un comportamiento lo bastante uniforme como nos gustaría, o bien, desconocemos nuevamente todos los factores que nos permitirían realizar una predicción, es decir, no tenemos a la mano un demonio de Laplace. Hacking lo expone con toda claridad: es más viable manejar certezas estadísticas que certezas absolutas, y con ello, el determinismo falla. Así lo expone

\footnotetext{
¿Cómo debía entender uno la estabilidad estadística en un universo laplaciano, en un universo en el que un espíritu convenientemente informado sería capaz de calcular todos y cada uno de los futuros sucesos partiendo de una exposición completa del estado de cosas en el universo y en un determinado momento? Laplace había dicho que la probabilidad es en parte el resultado de nuestro conocimiento y en parte el resultado de nuestra ignorancia. ${ }^{25}$
}

Por su parte, y como colofón, la mecánica cuántica, al estudiar el comportamiento de los eventos subatómicos, se encuentra imposibilitada para llevar a cabo una predicción satisfactoria empleando la mecánica newtoniana. Para llevar a cabo su cometido, introduce entonces dos ideas esenciales para percibir los eventos del microcosmos: la primer idea conlleva el derrumbamiento de la certeza -tan característica de la física clásica-, ${ }^{26}$ al encontrar que

24 Ibid. Popper. El Universo abierto. pp. 64-100.

25 Ibíd., Hacking. La domesticación del azar, p. 165.

26 Recordemos que un fenómeno aleatorio (fortuito o al azar) es un fenómeno empírico que se caracteriza por la propiedad de que, al observarlo bajo determinado conjunto de condiciones, no siempre se obtiene el mismo resultado, es decir, no existe regularidad determinista, sino que los diferentes resultados ocurren con regularidad estadística; esto lleva a decir que existen números entre los valores 0 y 1 que representan la frecuencia relativa con la que se observan ivs dic - nntes resultados en una serie de repeticiones independientes del fenómeno. Decimos entonces que la probabilidad de que ocurra el evento $\mathrm{A}$, denotada por $\mathrm{P}(\mathrm{A})$, con $\mathrm{A}$ elemento de un espacio muestral $\Omega$, es un valor que se encuentra entre los valores 0 y l. En términos probabílisticos, la certeza -o evento seguro- está dado por $P(\Omega)=1$, mientras que el evento imposible está dado por $\mathrm{P}(\mathrm{A})=0$. Más aún, si se tiene una serie muy grande de pruebas, se observa que el evento favorable puede producirse con una frecuencia próxima a su probabilidad (ley empírica 
la posición de una criatura atómica no puede determinarse con precisión en sitios oen tiempos definidos, siendo necesario introducir conceptos estadístico-probabilísticos para hacerlo, propiciando con ello que el manejo de la probabilidad sea un elemento fundamental para la comprensión y explicación del acontecer atómico y exaltando nuevamente la indeterminación. Veladamente, esto lleva a afirmar que en la naturaleza no existen leyes naturales, sino leyes estadísticas. ${ }^{27}$

La segunda idea no dejó de causar sorpresa al proponer que un observador, al estudiar un suceso atómico, ocasiona una perturbación en el desarrollo del fenómeno bajo estudio, llevándonos a una relación -tal vez directa-: fenómeno cuántico-observador, lo que inevitablemente nos regresa al subjetivismo.

El indeterminismo pues, retoma un nuevo impulso, al ser catapultado y establecido sin ambages por la postulación de los Principios de Complementariedad de Bohr y de Indeterminación o Principio de Incertidumbre de Heisenberg, que pueden enunciarse como:

Al querer asignar atributos físicos habituales a los objetos atómicos, como resulta evidente en el dilema planteado respecto a las propiedades corpusculares y ondulatorias de los electrones y fotones, nos enfrentamos con imágenes contrapuestas, cada una de las cuales se refiere a un aspecto esencial de la información empírica (de ellos obtenida). ${ }^{28}$ (Principio de Complementa-

del azar); así que, un suceso cuya probabilidad no difiere de I sino en una cantidad extremadamente pequeña $\left(=10^{100}\right)$, puede considerarse, efectivamente, como humanamente cierto.

27 Aquí no hay que perder de vista que las cuestiones estadísticas siempre son problemas de las probabilidades de las causas. En forma más clara, Borel arguye "...Es necesario decir algunas palabras acerca de la forma en que se plantean, con frecuencia, los problemas prácticos, siendo de la forma: "tal resultado, ise debió al azar o tuvo alguna causa? A esta pregunta, la teoría de probabilidades proporciona a menudo una respuesta que no habría que considerar como decisiva, pero que sería excesivo negarle todo valor". (Borel, E. (1996); Probabilidad y Errores: Colección Metronómica, Serie Metrología Técnica, Limusa Noriega Ed.; México. p 98.

28 Bohr, N. (1964); Física atómica y conocimiento humano; Aguilar, S. A. de Ediciones; España; p 49-50. Esto puede decir, de manera más simple, que los objetos no son ondas ni partículas, sino que asumen el papel de uno u otro objeto dependiendo de la observación que se lleve a cabo. Formalmente se enuncia: "Los objetos en la naturaleza no son partículas ni son ondas; un experimento o medición que resalte una de estas propiedades, lo hace necesariamente a expensas de la otra. ... La descripción cuántica de las propiedades de un sistema físico se expresa en términos de parejas de variables mutuamente complementarias. La precisión en la determinación de una de estas variables, necesariamente implica una imprecisión en la determinación de la otra." 
riedad). No es posible determinar la fijación de dos variables conjugadas q y p de una partícula con precisión ilimitada, es decir, Principio de Incertidumbre. ${ }^{29}$

La mecánica cuántica causó un hondo impacto en el pensamiento científico al proponer una insólita visión de la naturaleza después de subir al escenario científico el indeterminismo y la discontinuidad de los procesos naturales. Puso en evidencia la realidad que cotidianamente percibimos como continua, arguyendo que la continuidad solamente aparece en los niveles ordinarios de observación, ya que a niveles atómicos la naturaleza se muestra totalmente discreta, desapareciendo así la posibilidad de describir con toda precisión el comportamiento de los sistemas microscópicos. Esta propuesta causó una gran conmoción en la comunidad científica e impulsó una revolución conceptual que puso en entredicho la eficacia explicativa de las leyes de Newton.

De entre los presupuestos fundamentales para el desarrollo teórico cuántico destaca el Principio de Heisenberg, por ser el que abre la puerta al indeterminismo como elemento constituyente esencial de la nueva física. Tal principio consiste en enunciar que la naturaleza es azarosa en sí misma al esgrimir que el azar es una característica propia del mundo externo, negando con ello la existencia de un orden natural que se manifiesta en forma de leyes naturales.

Sin embargo, si bien esto puede ser admisible a nivel cuántico, en lo que respecta al nivel macroscópico no es del todo claro, puesto que la postura determinista ha soportado con firmeza los embates de la postura indeterminista, y lo ha conseguido gracias a su eficacia predictiva y su poder explicativo que dan cuenta del por qué de la ocurrencia natural, a tal grado que aún hoy sigue siendo la figura

(Saxon, D. S. (1968); Elementary Quantum Mechanics; Holden-Day, USA; pp 16-17).

29 Las variables conjugadas de una particula pueden ser, por ejemplo, la cantidad de movimiento $\Delta p$ y la posición $\Delta x$, o la energía $\Delta E$, y el tiempo $\Delta t$. En todo caso, se cumple que $\Delta q \Delta p \geq h / 2 \pi$. siempre; $h$ se denomina constante universal de Planck o "cuanto de acción", donde $h=6.625 \times 10^{-34}$ J.s 
emblemática del explicacionismo cientifico. Y es que la visión de algunos científicos que consideraron a la naturaleza como un gigantesco mecanismo donde era necesaria cierta secuencialidad en los eventos (la causa antecediendo al efecto) ha permeando variopintas teorías que han aparecido a lo largo de tres siglos, haciendo que de una u otra manera se sujetaran a la legislación newtoniana. Esta legislación introdujo el concepto de fuerza como el 'eslabonador' (incluso lógico) de los sucesos; sin duda esto fue lo que permitió que la fuerza se erigiera como la panacea explicativa del mundo natural. Aún perdura esta idea, y el autor encuentra que este concepto -o sus modalidades, como la teoría de los camposreúne los requisitos para continuar explicando el por qué de las cosas, y que cuando no logramos hacerlo así, caemos en problemas que parecen ser irresolubles. El estudio de los objetos atómicos, hasta este momento, es uno de esos problemas, y quizá por ello, la mecánica cuántica parece inclinarse por el indeterminismo.

Sinembargo,elproblemacentraldebeser, másqueeldeterminismo, la amplitud y validez de la relación causal, pues a fin de cuentas es ella la que aparece en las tesis deterministas e indeterministas. Y digo esto porque me parece que la tesis indeterminista no se puede sostener por sí misma aludiendo a la repetitividad de los eventos y apoyándose solamente en las regularidades estadísticas, pues a fin de cuentas, el hecho de que haya una regularidad estadística implica, de necesidad, una región de estabilidad que el sistema debe alcanzar en determinados momentos, y esto es una explicación determinista

Una cosa es clara: ni la ciencia ni la filosofía han demostrado de manera completa, objetiva y fríamente, si el determinismo -o más bien, la relación causal- vale o no en la naturaleza, pues ambas posturas tienen argumentos de mucha valía a favor y en contra. El determinismo ha sido fieramente apologizado y duramente vilipendiado, y en el mismo talante se encuentra el indeterminismo; pero considero que son creencias personales las que hacen que una persona se incline hacia un lado u otro. Por mi parte, me inclino más por el determinismo, sobre todo porque creo que existen leyes
C A
E
D
S
C
P 
naturales, así sea en los arcanos más profundos y oscuros del átomo, que son los que posibilitan el equilibrio y la estabilidad de los sistemas.

\section{Conclusiones}

Como se ha visto a lo largo del presente trabajo, desde que el ser humano comenzó a observar con mayor detenimiento su entorno, ha buscado dar una explicación del acontecer de las cosas. Para ello, ha recurrido a los dioses o a principios básicos que expliquen el por qué de los sucesos. Más aún, el desarrollo de la filosofía, y posteriormente de la física, posibilitó tener la impresión de que el funcionamiento de la naturaleza es equivalente al de un mecanismo, obligándola a admitir una relación de causa-efecto. Esta idea ensalzó algunos modelos exitosos debido a su elevada precisión en la predicción de los acontecimientos. Tal tipo de explicación ha recibido el mote de determinista.

Sin embargo, al encontrar un fenómeno que sale de la potestad de la teoría explicativa, ha hecho dudar a varios pensadores y científicos si el determinismo vale o no, lo que ha llevado el pensamiento hacia el otro extremo, es decir, hacia el indeterminismo.

Una gran controversia entre los apologistas de una u otra doctrina se ha levantado. Por la filosofía descuellan, sin duda alguna, los indeterministas Hume y Popper, quienes con su capacidad intelectual y agudo ingenio han cuestionado fuertemente las tesis deterministas.

Pero no sólo están los cuestionamientos filosóficos, pues en la ciencia también se han suscitado tales polémicas, teniéndose de un lado dos grandes contendientes que parecen dar la razón al determinismo científico: la mecánica clásica y la teoría de la relatividad. Del otro lado, está la mecánica cuántica, un rival de gran fortaleza conceptual que parece inclinarse por el indeterminismo.

Los argumentos de ambos bandos son sólidos, y hay que analizarlos concienzudamente para llegar a determinar con mayor 
claridad si el determinismo en la mecánica cuántica, sobre todo en lo relativo al principio de incertidumbre, es válido o no. Para ello, hay que desenmarañar la madeja de argumentos deterministas e indeterministas relacionados con él, porque ambos rivales cuentan con grandes logros que presumir y argumentos de mucho peso, a favor o en contra. La moneda pues, está en el aire.

\section{BibLIOGRAFÍA}

Beuchot, M. (1987); Conocimiento, Causalidad y Metafísica; Universidad Veracruzana, Xalapa; México.

Bird, A. (1998).pdf: Philosophy of Science; Fundamentals of Philosophy.

Series Editor: John Shand. University of Edinburgh. Routledge.

Taylor \& Francis Group.

Borel, E. (1996); Probabilidady Errores; Colección Metronómica, Serie Metrología Técnica, Limusa Noriega Ed.

Earman, J. (1986).pdf: A primer on determinism; D. Reidel Publishing Company; Netherlands.

Enciclopedia Hispánica. Vol. 5; Encyclopaedia Britannica Publishers, Inc. USA. 1995-1996.

Ferrater Mora, J. (1964).pdf: Diccionario de filosofía. Tomo I: A-K; Ed. Sudamericana. Buenos Aires.

Gamow, G. (1971): Biografía de la física; Biblioteca General Salvat; Salvat Editores; España, 1971.

Hacking, I (1995); La domesticación del azar (La erosión del determinismo y el nacimiento de las ciencias del caos); Ed. Gedisa. España.

Lagemann, R. T. (1963): Ciencia Física. Orígenes y Principios; Unión

Tipográfica Editorial Hispano Americana. México, 1968.

Mach, R. (1977); Física para poetas; Siglo XXI Editores. México

Marías, J. (1941): Historia de la filosofía. 30 Ed.; Biblioteca de la Revista de Occidente. Madrid. 1978.

Martínez, Sergio F. (2001): De los efectos a las causas. Sobre la historia de los patrones de explicación científica; UNAM-Paidós.

More, L. T. (1934): Isaac Newton, A Biography; Dover. USA.

Newton, I. (1686): Escolio de los Philosophiae naturalis principa 
mathematica; en: Einstein, A., Grünbaum, A., Eddington, A. S. y otros (1978): La teoría de la relatividad. Sus origenes e impacto sobre el pensamiento moderno; Selección de Williams, L. Pearce; Alianza Editorial. Madrid. 1978.

(1729).pdf: Mathematical principles of natural philosophy and his system of the world. Vols. II \& III: The system of the world. University of California Press. USA.

(1987): Principios matemáticos de la filosofía natural, 2. Libro II y Libro III; Alianza Editorial, S. A.; Madrid.

Platón; Diálogos de Platón. Teetetes o de la ciencia; 15a . Edición; Editorial Porrúa, S. A.; México, 1975.

Popper, K. R. (1982): Conocimiento Objetivo. Un enfoque evolucionista; Ed. Tecnos. Madrid

(1996): El universo abierto. Un argumento a favor del indeterminismo. Post Scriptum a la Lógica de la Investigación Científica. Vol. II.; Ed. Tecnos. Madrid. (1992): Un mundo de propensiones; Ed. Tecnos. Madrid.

Psillos, S. (2007).pdf: Philosophy of science A-Z; Edinburgh University Press; G. B.

Saxon, D. S. (1968); Elementary Quantum Mechanics; Holden-Day, USA.

Stroud, B. (1995); Hume; Colección Filosofía Contemporánea; Instituto de Investigaciones Filosóficas, UNAM; México.

Symon, K. R. (1977): Mecánica; Colección Ciencia y Técnica. Ed. Aguilar; España.

Referencias

Enciclopedia Encarta; Microsoft 2000.

Enciclopedia Católica. Libre voluntad. http://ecaciprensa.com/// librevoluntad.htm

http://es.wikipedia.org/wiki/Estoicismo

http://www.La construcción científica de la realidad_Determinismo

e indeterminismo_El postulado de la objetividad.htm

http://it.wikipedia.org/wiki/Ecfanto_di_Siracusa

http://es.wikipedia.org/wiki/Galileo_Galilei 
http://es.wikipedia.org/wiki/Causa (desambiguaci\%C3\%B3n) http:/www.biografiasyvidas.com/biografia//leucipo.htm http://www.monografias.com/trabajosl6/nicolas-copernico/ nicolas-copernico.shtml $\#$ COSMOL http://www.dpye.iimas.unam.mx/PresentacionesJornadas2007/ IgnacioMendez.pdf. 
